\title{
BLIND IDENTIFICATION OF DISTRIBUTED ANTENNA SYSTEMS WITH MULTIPLE CARRIER FREQUENCY OFFSETS
}

\author{
Yuanning Yu, Athina P. Petropulu and H. Vincent Poor ${ }^{+}$ \\ Electrical \& Computer Engineering Department, Drexel University \\ ${ }^{+}$School of Engineering and Applied Science, Princeton University
}

\begin{abstract}
In spatially distributed multiuser antenna systems, the received signal contains multiple carrier-frequency offsets (CFOs) arising from mismatch between the oscillators of transmitters and receivers. This results in a time-varying rotation of the data constellation, which needs to be compensated at the receiver before symbol recovery. In this paper, a new approach for blind CFO estimation and symbol recovery is proposed. The received base-band signal is over-sampled, and its polyphase components are used to formulate a virtual Multiple-Input Multiple-Output (MIMO) problem. By applying blind MIMO system estimation techniques, the system response can be estimated and decoupled versions of the user symbols can be recovered, each one of which contains a distinct CFO. By applying a decision feedback Phase Lock Loop (PLL), the CFO can be mitigated and the transmitted symbols can be recovered. The estimated MIMO system response provides information about the CFOs that can be used to initialize the PLL, speed up its convergence, and avoid ambiguities usually linked with PLL.

keywords-Multi-user Systems, Distributed Antenna Systems, Carrier Frequency Offset, Blind MIMO System Identification
\end{abstract}

\section{BACKGROUND}

In both wireless and wireline communication systems, received signals are often corrupted by carrier-frequency offsets (CFOs), due to Doppler shift and/or local oscillator drift. The CFO causes a time-varying rotation of the data symbols, and thus before symbol recovery, it must be estimated and accurately compensated for by the receiver. The CFO can be estimated via the use of pilots symbols; however, even a small error in this estimation tends to cause large data recovery errors. This necessitates transmission of pilot symbols rather often. In single user systems, or in multiuser systems where the transmitters are physically connected to the same oscillator, there is only one CFO that needs to be estimated. This is typically done via a decision feedback Phase Lock Loop (PLL) at the receiver. The PLL is a closed-loop feedback control system that can adaptively track both frequency and phase offsets between the equalized signals and the reference constellation. However, depending on the constellation used during transmission, the PLL can have an $M$-fold symmetric ambiguity, and thus it has limited CFO acquisition range; e.g., $\left|f_{k}\right|<1 / 8$ for 4QAM signals. Moreover, the PLL require a long convergence time. To solve these problems, several methods have been proposed [3], [5], [6], [9] [11] that allow for blind estimation of the CFO and symbols using the second-order cyclo-stationary statistics of the over-sampled received signal. Blind CFO estimation has also been studied in the context of orthogonal

This work was supported by the U. S. National Science Foundation under Grants ANI-03-38807, CNS-06-25637 and CNS-04-35052. frequency-division multiplexing (OFDM) systems, where the CFO destroys the orthogonality between the carriers (see [4] and the references therein).

In a spatially distributed multiuser antenna system where data are transmitted simultaneously from multiple antennas, the received signal contains multiple CFOs, one for each transmit antenna. A PLL does not work in this case as there is no single frequency to lock onto. The literature on estimation of multiple CFOs is rather sparse. In [8], multiple CFOs were estimated by using pilot symbols that were uncorrelated among the different users. To account for multiple offsets, [10] proposed that multiple nodes transmit the same copy of the data with an artificial delay at each node. The resulting system was modeled as a convolutive single-input/singleoutput (SISO) system with time-varying system response caused by the multiple CFOs. A minimum mean-square error (MMSE) decision feedback equalizer was used to track and equalize the channel and to recover the input data. Training symbols were required in order to obtain a channel estimate, which was used to initialize the equalizer.

In this paper, a new approach to blind CFO estimation and symbol recovery is proposed. The received base-band signal is oversampled, and its polyphase components are used to formulate a virtual MIMO problem. By applying blind MIMO system estimation techniques, the system response can be estimated, and decoupled versions of the user symbols can be recovered, each one of which contains a distinct CFO. By applying a PLL, the CFO can be mitigated and the transmitted symbols can be recovered. The estimated MIMO system response provides information about the CFOs that can be used to initialize the PLL, speed up its convergence, and avoid ambiguities usually linked with PLLs.

\section{SYSTEM MODEL}

We consider a distributed antenna system, where $K$ users transmit simultaneously to a base station. Narrow-band transmission is assumed here, where the channel between any user and the base station is frequency non-selective. In addition, quasi-static fading is assumed, i.e., the channel gains remain fixed during the packet length. The continuous-time base-band received signal $y(t)$ can be expressed as

$$
y(t)=\sum_{k=1}^{K} a_{k} x_{k}\left(t-\tau_{k}\right) e^{j 2 \pi F_{k} t}+w(t),
$$

where $a_{k}$ represents the effect of channel fading between the $k$-th user and the base station and also phase offset; $\tau_{k}$ is the delay associated with the path between the $k$-th user and the base station; $F_{k}$ is the frequency offset of the $k$-th user and $w(t)$ represents noise; 
$x_{k}(t)$ denotes the transmitted signal of user $k$ :

$$
x_{k}(t)=\sum_{i} s_{k}(i) p\left(t-i T_{s}\right),
$$

where $s_{k}(i)$ is the $i$-th symbol of user $k ; T_{s}$ is the symbol period; and $p(t)$ is a pulse function with support $\left[0, T_{s}\right]$.

Our objective is to obtain an estimate of $\mathbf{s}(i)=$ $\left[s_{1}(i), \ldots, s_{K}(i)\right]^{T}$ in the form

$$
\hat{\mathbf{s}}(i)=\hat{\Lambda} \mathbf{P}^{T} \mathbf{s}(i)
$$

where $\mathbf{P}$ is a column permutation matrix and $\hat{\boldsymbol{\Lambda}}$ a constant diagonal matrix. These are considered to be trivial ambiguities, and are typical in any blind problem.

\section{FORMATION OF THE MIMO PROBLEM}

The received signal $y(t)$ is sampled at rate $1 / T=P / T_{s}$, where the over-sampling factor $P \geq K$ is an integer. In order to guarantee that all the users' pulses overlap at the sampling times, the over-sampling period should satisfy: $T_{s} / P \geq \tau_{k}, k=1, \ldots K$. Or, in other words, the over-sampling factor $\bar{P}$ is upper bounded by $T_{s} / \min \left\{\tau_{1}, \ldots, \tau_{K}\right\}$.

Let $t=i T_{s}+m T, m=1, \ldots, P$, denote the sampling times. The over-sampled received signal can be expressed as

$$
\begin{aligned}
& y_{m}(i)=y\left(i T_{s}+m T\right) \\
= & \sum_{k=1}^{K} a_{k} e^{j 2 \pi f_{k}\left(i+\frac{m}{P}\right)} x_{k}\left(\left(i+\frac{m}{P}\right) T_{s}-\tau_{k}\right)+w\left(\left(i+\frac{m}{P}\right) T_{s}\right) \\
= & \sum_{k=1}^{K} a_{k} e^{j 2 \pi f_{k}\left(i+\frac{m}{P}\right)} s_{k}(i) p\left(\frac{m}{P} T_{s}-\tau_{k}\right)+w\left(i T_{s}+\frac{m}{P} T_{s}\right) \\
= & \sum_{k=1}^{K} a_{m, k}\left(s_{k}(i) e^{j 2 \pi f_{k} i}\right)+w\left(i+\frac{m}{P}\right), m=1, \ldots, P,
\end{aligned}
$$

where $f_{k}=F_{k} T_{s}$ is the normalized frequency offset between the $k$-th user and the base station, and the typical element of the virtual MIMO channel matrix $\mathbf{A}$ is given by

$$
a_{m, k}=a_{k} e^{j 2 \pi \frac{f_{k}}{P} m} p\left(\frac{m}{P} T_{s}-\tau_{k}\right) .
$$

Define the following: $\mathbf{y}(i) \triangleq\left[y_{1}(i), \ldots, y_{P}(i)\right]^{T}$; $\mathbf{A}=\left\{a_{m, k}\right\}$, a tall matrix of dimension $P \times K$; $\tilde{\mathbf{s}}(i) \triangleq\left[s_{1}(i) e^{j 2 \pi f_{1} i}, \ldots, s_{K}(i) e^{j 2 \pi f_{K} i}\right]^{T} ; \quad$ and $\quad \mathbf{w}(i) \triangleq$ $\left[w\left(i+\frac{1}{P}\right), \ldots, w\left(i+\frac{P}{P}\right)\right]^{T}$. Then, (4) can be written in matrix form as

$$
\mathbf{y}(i)=\mathbf{A} \tilde{\mathbf{s}}(i)+\mathbf{w}(i) .
$$

We could use the training based method of [8] to solve the MIMO system (4). That method assumes that the pilot symbols of different users are uncorrelated. The CFOs are obtained by searching for the location of a peak in the cross-correlation between the Discrete-Time Fourier Transform (DTFT) of a pilot sequence and that of the received signal.

In the following we show how to estimate CFOs and recover the transmitted signals in a bind fashion, i.e., without the need for pilot symbols. The advantage of a blind approach is bandwidth efficiency since no bandwidth is wasted transmitting pilot symbols.

\section{BLIND CHANNEL ESTIMATION AND COMPENSATION OF THE CFOS}

Let us make the following assumptions.

- A1) For each $m=1, \ldots, P, w_{m}($.$) is a zero-mean Gaus-$ sian stationary random processes with variance $\sigma_{w}^{2}$, and is independent of the channel inputs.

- A2) For each $k$, the sequence $s_{k}(i)$ is a zero mean with independent and identically distributed (i.i.d.) elements having nonzero kurtosis; i.e., $\gamma_{s_{k}}^{4}=\operatorname{Cum}\left[s_{k}(i), s_{k}^{*}(i), s_{k}(i), s_{k}^{*}(i)\right] \neq 0$. The sequences $s_{k}$ 's are also mutually independent.

- A3) The over-sampling factor $P$ is no less than $K$.

Under assumption (A2), it is easy to verify that the rotated input signals $\tilde{s}_{k}($.$) are also zero mean and i.i.d with nonzero kurtosis. Also,$ the $\tilde{s}_{k}(i)$ 's are mutually independent for different $k$ 's. Assumption (A3) guarantees that the virtual MIMO channel matrix A in (6) has full rank with probability one. If the delays of users are randomly distributed in the interval $\left[0, T_{s} / P\right)$, then each row of the channel matrix can be viewed as having been drawn randomly from a continuous distribution so that the channel matrix has full rank with probability one.

One can apply any blind source separation algorithm (e.g., [1],[2] or [7] ) to obtain

$$
\hat{\mathbf{A}} \triangleq \mathbf{A P} \Lambda
$$

Subsequently, using a least-squares equalizer we can obtain an estimate of the de-coupled signals $\tilde{\mathbf{s}}(i)$, within permutation and scalar ambiguities as

$$
\hat{\tilde{\mathbf{s}}}(i)=\left(\hat{\mathbf{A}}^{H} \hat{\mathbf{A}}\right)^{-\mathbf{1}} \hat{\mathbf{A}}^{\mathbf{H}} \mathbf{y}(i)=e^{j \operatorname{Arg}\{-\boldsymbol{\Lambda}\}}|\boldsymbol{\Lambda}|^{-1} \mathbf{P}^{T} \tilde{\mathbf{s}}(i) .
$$

Without loss of generality we can assume that the transmitted signal has unit power. Then, on denoting by $\theta_{k}$ the $k$-th diagonal element of $\operatorname{Arg}\{\boldsymbol{\Lambda}\}$, the $j-$ th separated input signal can be expressed as

$$
\hat{\tilde{s}}_{k}(i)=s_{k}(i) e^{j\left(-\theta_{k}+2 \pi f_{k} i\right)} .
$$

In order to recover the transmitted signals, we still need to mitigate the effect of CFO in each decoupled signal. This can be done via a PLL. By using the decoupled signals as inputs, and the constellation used in transmission as a reference, the PLL can effectively mitigate the $\mathrm{CFO}$ by minimizing the feedback error, which is calculated based on the distance of the recovered signal and the closest valid constellation point. However, depending on the constellation used in transmission, there is a four-fold symmetric ambiguity for MQAM signals, or $M$-fold symmetric ambiguity for MPSK signals. For example, for 4QAM signals, and an initial CFO value of $f_{k}=0$, the effective tracking range for $f_{k}$ is $\left|f_{k}\right|<1 / 8$. Moreover, depending on the value of the CFO, the PLL generally needs a long convergence time, during which the input signals are not correctly recovered.

Next we will show that by exploring the structure of the virtual channel matrix, we can obtain an estimate of the CFOs, which can then be used to initialize the PLL. By doing this, we can prevent the symmetric ambiguity problem and enlarge the effective tracking range of the PLL from $\left|f_{k}\right|<1 / 8$ to $\left|f_{k}\right|<1 / 2$. Also, the convergence time of the PLL can be greatly reduced. 
By taking the phase of the estimated channel matrix $\hat{\mathbf{A}}$, we obtain

$$
\Psi=\operatorname{Arg}\{\hat{\mathbf{A}}\}=\left(\begin{array}{ccc}
\frac{2 \pi f_{1}}{P}+\phi_{1} & \ldots & \frac{2 \pi f_{K}}{P}+\phi_{K} \\
\vdots & \ddots & \vdots \\
\frac{2 \pi f_{1} P}{P}+\phi_{1} & \ldots & \frac{2 \pi f_{K} P}{P}+\phi_{K}
\end{array}\right) \mathbf{P}
$$

where $\phi_{k}=\operatorname{Arg}\left\{a_{k}\right\}+\theta_{k}$, which accounts for both the phase of $a_{k}$ and the estimated phase ambiguity in 9.

By applying linear fitting on the $j$-th column of $\Psi$ we obtain the least squares estimate of $f_{j}$ as

$$
\hat{f}_{j}=\frac{P}{2 \pi} \frac{P\left(\sum_{p=1}^{P} p \Psi_{p, j}\right)-\left(\sum_{p=1}^{P} p\right)\left(\sum_{p=1}^{P} \Psi_{p, j}\right)}{P\left(\sum_{p=1}^{P} p^{2}\right)-\left(\sum_{p=1}^{P} p\right)^{2}} .
$$

We can write $\hat{f}_{j}=f_{j}+\epsilon_{j}$ where $\epsilon_{j}$ represents estimation error.

On noting that the de-coupled signals $\hat{\tilde{s}}_{j}(i)$ in (9) are shuffled in the same manner as the estimated CFOs in 111, we can use the estimated CFOs to compensate for the effects of CFO in the decoupled signals $(9)$ and thereby obtain estimates of the input signals as

$$
\hat{\mathbf{s}}(i)=e^{j \operatorname{Arg}\{-\boldsymbol{\Lambda}\}} \mathbf{P}^{T} \mathbf{s}(i) .
$$

Due to errors in the channel estimates, we can only compensate for most, but not all, of the CFO effects in (9) and so we can write

$$
\hat{s}_{k}(i)=s_{k}(i) e^{j\left(-\theta_{k}-2 \pi \epsilon_{k} i\right)} .
$$

By subsequently applying a PLL to $\hat{s}_{j}(i)$, we can further mitigate the effects of the residual CFO $\epsilon_{k}$. For 4QAM signals, as long as $\left|\epsilon_{k}\right|<1 / 8$, the residual CFO can be effectively removed by the PLL. The initial CFO estimator (11) can prevent the symmetric ambiguity of the PLL, and can also greatly reduce the convergence time of the PLL. From (10), we can see that the CFO estimator will achieve full acquisition range for the normalized CFO.

\section{SIMULATION RESULTS}

In this section, we verify the validity of the proposed method via simulations, under the following assumptions. The channel coefficients $a_{k}, k=1, \ldots, K$ are zero-mean Gaussian random variables. The waveform $p(\cdot)$ is a Hamming window. The delays, $\tau_{k}, k=1, \ldots, K$ are uniformly distributed in the range $\left[0, T_{s} / P\right)$. The input signals are 4QAM signals.

The blind source separation algorithm used here is the JADE method, which was downloaded from http://www.tsi.enst.fr/c̃ardoso/guidesepsou.html

First we show results for a two-user systems with $f_{1}=$ $-0.1552, f_{2}=0.4335, a_{1}=0.3173-0.6483 i, a_{2}=0.1625+$ $0.5867 i$, with $\mathrm{SNR}=20 d B$, and $N=1024$. In Fig. 1 we show the polyphase outputs $y_{1}, y_{2}$. Due to the mixing and the CFOs no obvious constellation is visible. In Fig. 2] we show the de-coupled signals $\hat{\tilde{s}}_{k}, k=1,2$ right after JADE. Although still rotated by the CFOs, two signals $\tilde{s}_{k}, k=1,2$ are clearly separated. In Fig. 3 we show the recovered input signals $\hat{s}_{k}, k=1,2$, where we can see that after compensating for the effect of CFOs the constellations are recovered.

Next we show estimation results averaged over 300 independent channel runs, and 20 Monte-Carlo runs for each channel. For each channel case, the coefficients $a_{k}, k=1,2$ were generated randomly, and the continuous CFOs where chosen randomly in the range $\left[-\frac{1}{2 T_{s}}, \frac{1}{2 T_{s}}\right)$. The delays, $\tau_{k}, k=1, \ldots, K$ where chosen uniformly in the range of $\left[0, T_{s} / P\right)$. The transmitted signal was 4QAM.

The performance of both the pilot-based method and the proposed method at different data lengths and with SNR set to $30 \mathrm{~dB}$ is shown in Fig. 4 For the pilot-based method, each user transmits a pilot sequence of length 32 , and the pilots are random sequences uncorrelated between different users. Fig. 4 shows the mean-square error (MSE) for the CFO estimate (11) for different values of the over-sampling factor $P$. The MSE is calculated usings $\frac{1}{K} \sum_{k=1}^{K}\left[\left(\hat{f}_{k}-f_{k}\right)\right]^{2}=\frac{1}{K} \sum_{k=1}^{K}\left[\left(\hat{F}_{k}-F_{k}\right) T_{s}\right]^{2}$. We can see that by increasing $P$ we can improve the estimation accuracy. Fig. 5 shows the Bit Error Rate (BER) for different values of $P$. For both blind and training methods, the BER is calculated based on the recovered signals after the PLL. As expected, the BER performance also improves by increasing $P$. The proposed method appears to work well even for short data length.

Next we show the performance of both methods at various noise levels. We use packet length $N=1,024$. In Fig. 6 we show the MSE of the blind CFO estimator (11) as well as that of the training based method. We can see that by increasing $P$ we improve estimation accuracy. In Fig. 7 we show the BER performance after the PLL for both blind and training based methods. We see that the proposed blind method has almost the same performance as the training based method for SNR values lower than $20 \mathrm{~dB}$, while the training based method can achieve better BER performance for higher values of SNR.

\section{CONCLUSION}

In this paper we have proposed a novel blind approach for identification of a distributed multiuser antenna system with multiple CFOs. By over-sampling of the received base-band signal, the MISO problem is converted into a MIMO one. Blind MIMO system estimation then yields the system response, and MIMO input recovery yields the decoupled transmitted signals, each one containing a CFO. By exploring the structure of the MIMO system response we obtain a coarse estimate of the CFOs, which is then combined with a decision feedback PLL to compensate for the CFOs in the decoupled transmitted signals. The proposed blind method has full acquisition range for normalized CFOs.

\section{REFERENCES}

[1] T. Acar, Y. Yu and A. P. Petropulu, "Blind MIMO system estimation based on PARAFAC decomposition of higher order output tensors" IEEE Trans. Signal Process., Vol. 54, No. 11, pp. 4156 - 4168, Nov. 2006

[2] J. F. Cardoso and A. Souloumiac, "Blind beamforming for nonGaussian signals," IEE Proceedings: Radar and Signal Process., Vol. 140, No. 6, pp. 362-370 Dec. 1993.

[3] P. Ciblat, P. Loubaton, E. Serpedin and G. B. Giannakis, "Performance analysis of blind carrier frequency offset estimators for noncircular transmissions through frequency-selective channels," IEEE Trans. Signal Process., Vol. 50, No. 1, pp. 130 - 140, Jan. 2002.

[4] T. Roman, S. Visuri and V. Koivunen, "Blind frequency synchronization in OFDM via diagonality criterion," IEEE Trans. Signal Process., Vol. 54, No. 8, pp. 3125 - 3135, Aug. 2006.

[5] M. Ghogho, A. Swami and T. Durrani, "On blind carrier recovery in time-selective fading channels," in Proc. 33rd Asilomar Conf. Signals, Systems and Computers, Vol. 1, pp. 243-247, Pacific Grove, CA, Oct. 1999, 
[6] F. Gini and G. B. Giannakis, "Frequency offset and symbol timing recovery in flat-fading channels: A cyclostationary approach," IEEE Trans. Commun., Vol. 46, No. 3, pp. 400 - 411, Mar. 1998.

[7] L. de Lathauwer, B. de Moor and J. Vandewalle, "Independent component analysis and (simultaneous) third-order tensor diagonalization," IEEE Trans. Signal Process., Vol. 49, No. 10, pp. 2262-2271, Oct. 2001

[8] F. Prihoda, E. Garbarine and A. P. Petropulu, "Resolving wireless collisions in random access networks," Proc. 2006 Asilomar Conf. Signals, Systems and Computers, Pacific Grove, CA, Oct. 2006.

[9] K. E. Scott and E. B. Olasz, "Simultaneous clock phase and frequency offset estimation," IEEE Trans. Commun., Vol. 43, No. 7, pp. 2263 2270, July 1995.

[10] D. Veronesi and D. L. Goeckel, "Multiple frequency offset compensation in cooperative wireless systems," Proc. IEEE Global Telecommun. Conf., San Francisco, CA, Nov. 2006.

[11] Y. Wang, P. Ciblat, E. Serpedin and P. Loubaton, "Performance analysis of a class of nondata-aided frequency offset and symbol timing estimators for flat-fading channels," IEEE Trans. Signal Process., Vol. 50, No. 9, pp. 2295- 2305, Sept. 2002.
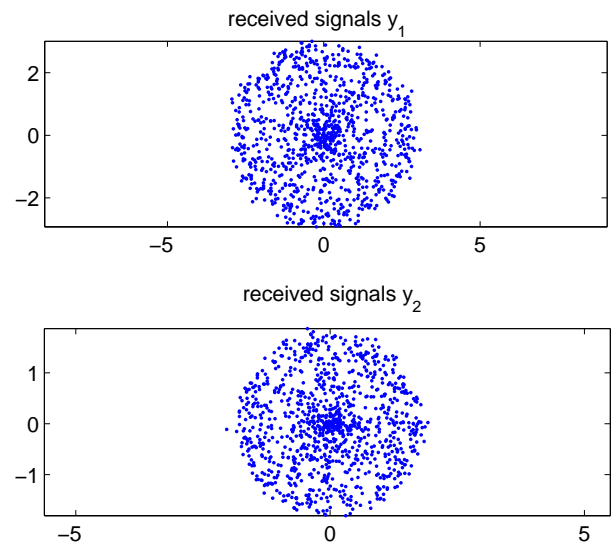

Fig. 1. Received mixing signal y
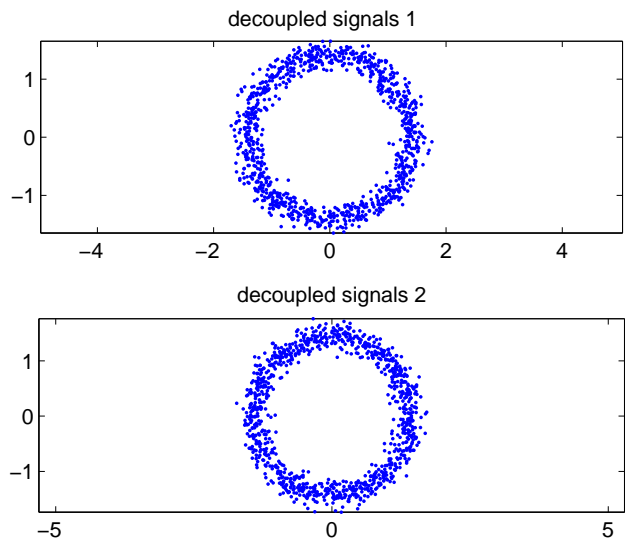

Fig. 2. De-coupled inputs $\hat{\tilde{\mathbf{s}}}$
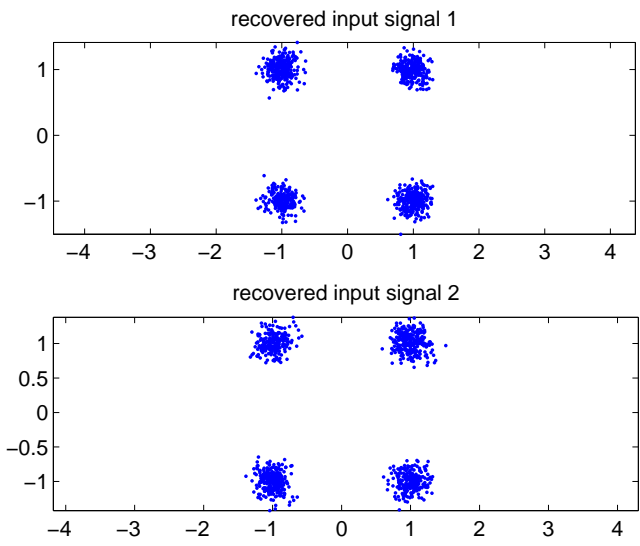

Fig. 3. Recovered input signals $\hat{\mathbf{s}}$ with $P=2$

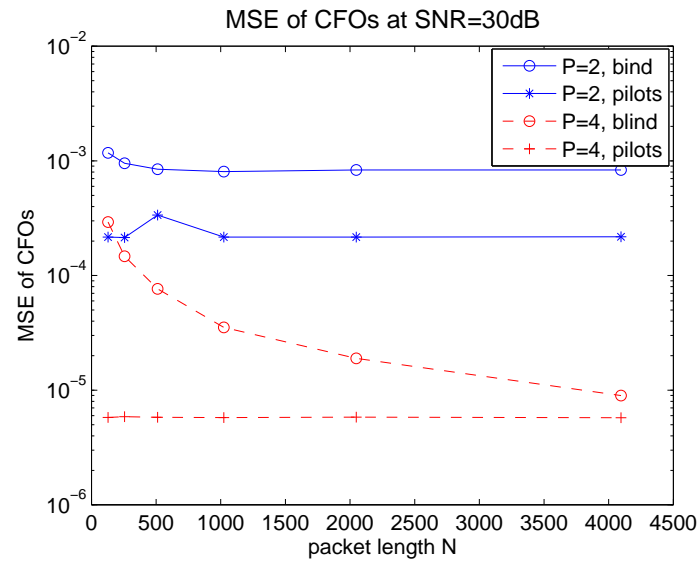

Fig. 4. MSE of CFOs vs $N$ for $\mathrm{K}=2$, with $\mathrm{SNR}=30 \mathrm{~dB}$, 4QAM

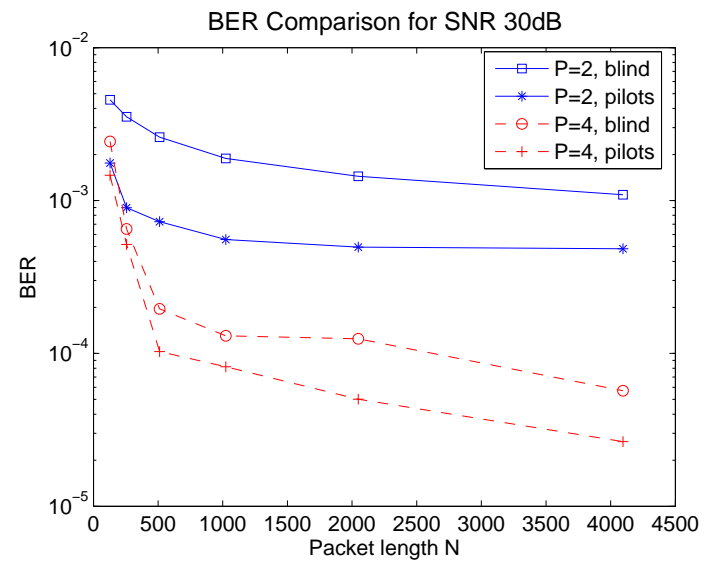

Fig. 5. BER vs $N$ for $\mathrm{K}=2$, with $\mathrm{SNR}=30 \mathrm{~dB}, 4 \mathrm{QAM}$ 


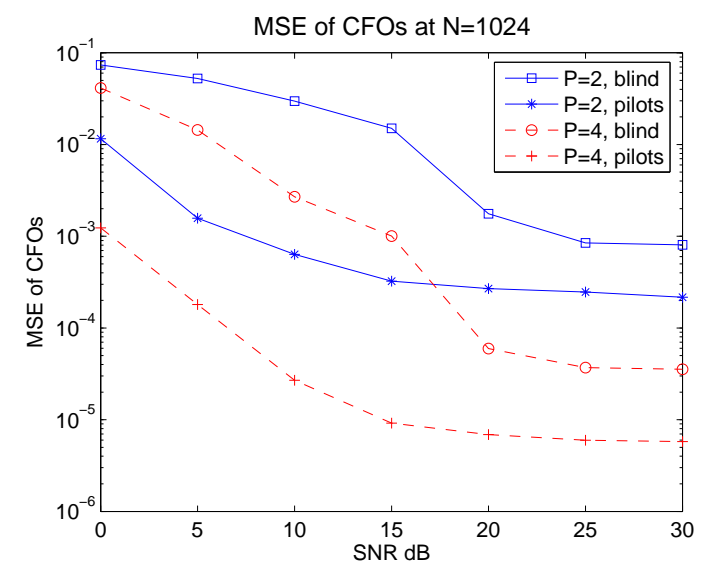

Fig. 6. MSE of CFOs vs SNR for $\mathrm{K}=2$, 4QAM

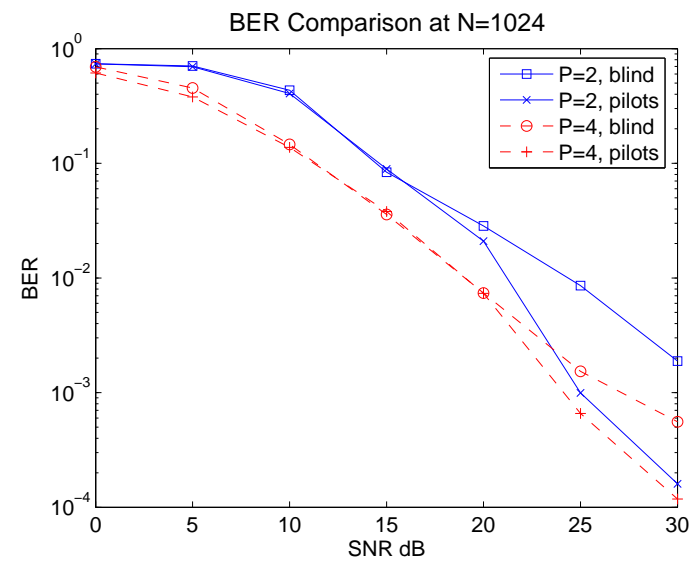

Fig. 7. BER vs $\mathrm{SNR}$ for $\mathrm{K}=2$, 4QAM, $\mathrm{T}=1024$ 